\title{
THE STRUCTURE OF NORMAL INVERSE SEMIGROUPS
}

\author{
by G. B. PRESTON
}

(Ruceived 6th August, 1955)

1. Introduction. In a recent paper [1] we showed that there is a $(1,1)$-correspondence between the homomorphisms of an inverse semigroup $S$ and its normal subsemigroups. The normal subsemigroup of $S$ corresponding to and determining the homomorphism $\mu$ of $S$ is the inverse image under $\mu$ of the set of idempotents of $S_{\mu}$, and is called the kernel of the homomorphism $\mu$. The inverse image of each idempotent of $S \mu$ is itself an inverse semigroup [1], and each such inverse semigroup is said to be a component of the normal subsemigroup determined by $\mu$.

A semigroup $S$ is said to be a band of semigroups of type $T$ (A. H. Clifford [2]), if $S$ is the class sum of a set $\left\{S_{\alpha} ; \alpha \epsilon J\right\}$ of mutually disjoint subsemigroups $S_{\alpha}$, each of type $T$, such that for any $\alpha, \beta \epsilon J$, there is a $\gamma \in J$ for which $S_{\alpha} S_{\beta} \subseteq S_{\gamma}$. If we write $\alpha \beta=\gamma$, then $J$ becomes a semigroup of idempotents. If $J$ is a commutative semigroup, that is, if $J$ is a semilattice, then $S$ is called a semilattice of semigroups of type $T$. This is equivalent to the condition that for any $\alpha, \beta \epsilon J, S_{\alpha} S_{\beta}$ and $S_{\beta} S_{\alpha}$ are both contained in the same $S_{\gamma}$.

If $S$ is an inverse semigroup, and $\mu$ is a homomorphism of $S$, then $S \mu$ is an inverse semigroup ([1], Theorem 1). Since the idempotents of $S \mu$ therefore form a semilattice, it is clear that any normal inverse semigroup is a semilattice of inverse semigroups. Conversely it is also clear that an inverse semigroup $N$ which is the union of the disjoint inverse semigroups $N_{\alpha}$, can be a normal inverse subsemigroup, with the $N_{\alpha}$ as its components, of some inverse semigroup $S$, if the $N_{\alpha}$ form a semilattice. It is sufficient to take $S=N$. Thus an inverse semigroup $N$ which is the union of the disjoint inverse semigroups $N_{\alpha}$ can be the kernel of some homomorphism of an inverse semigroup, with the given $N_{\alpha}$ as its components, if and only if $N$ is a semilattice of inverse semigroups, the elements of the semilattice being the $N_{\alpha}$. We show (Theorem 1) that if $E$ is the set of idempotents of $N$, and $E_{\alpha}$ is the set of idempotents of $N_{\alpha}$, then this necessary and sufficient condition may be replaced by the weaker condition that $E$ is a semilattice with elements $E_{\alpha}$. A special case of Theorem 1 provides an alternative proof of a result of Clifford [3], also proved by A. E. Liber [4], that semigroups with relative inverses in which the idempotents commute are semilattices of groups.

We then show (Theorem 2) that any semigroup which is a semilattice of inverse semigroups is necessarily an inverse semigroup. This generalises and also provides an alternative proof of the result of $R$. Croisot that a semilattice of groups is an inverse semigroup (Croisot [5], p. 375, proved independently by Clifford [2], Theorem 8, and also proved by W. D. Munn and R. Penrose [6]).

Finally we investigate the structure of normal inverse semigroups, that is, semilattices of inverse semigroups, and show that the structure of such a semigroup $S$ is determined to within isomorphism by the semilattice, the elements of the semilattice, and certain right translations of $S$. The set of all such semilattices with associated right translations which determine $S$ forms a set of complete invariants for $S$ (Theorem 3 ). A set of complete invariants has already been found by Clifford [3] for a semilattice of groups. Our conditions provide another set of complete invariants for this special case, more complicated however than those of Clifford. 
2. Normal inverse semigroups. An inverse semigroup is a semigroup $S$ in which for any $a \in S$ the equations $a x a=a$ and $x a x=x$ have a unique common solution $x \in S$. This unique solution is denoted by $a^{-1}$. It then follows if we put $a a^{-1}=e$ and $a^{-1} a=f$, that

$$
\begin{gathered}
e^{2}=e, \quad f^{2}=f, \\
e a=a=a f, \\
f a^{-1}=a^{-1}=a^{-1} e .
\end{gathered}
$$

$a^{-1}$ is the inverse of $a, e$ is the left unit of $a, f$ is the right unit of $a . e$ and $f$ are unique with the above properties in relation to $a$. The inverse of $a^{-1}$ is $a$, its left unit is $f$, its right unit is $e$. For any $a, b \in S,(a b)^{-1}=b^{-1} a^{-1}$. Any two idempotents of $S$ commute.

A discussion of alternative definitions of inverse semigroups is given by Munn and Penrose in [6]. The definition above was first given by Liber [4]. For other definitions and a derivation of the above properties see [1] and V. V. Vagner's paper [7].

Theorem 1. Let $N$ be an inverse semigroup which is the union of the disjoint inverse semigroups $N\left(E_{\alpha}\right)$ for $\alpha \epsilon J$. $E_{\alpha}$ denotes the set of idempotents of $N\left(E_{\alpha}\right)$. Let $E$ be the set of idempotents of $N$. Then $N$ is a semilattice of inverse semigroups, with the $N\left(E_{\alpha}\right)$ as the elements of the semilattice, if and only if $E$ is a semilattice of semilattices, the $E_{\alpha}$ being the elements of this semilattice.

Proof. That the condition is necessary follows from the fact the product of two idempotents of an inverse semigroup is an idempotent.

To show that it is sufficient, let $\alpha, \beta$ be any two elements of $J$. Then there is a $\gamma \in J$ such that

$$
E_{\alpha} E_{\beta}\left(=E_{\beta} E_{\alpha}\right) \subseteq E_{\gamma} .
$$

Let $n \in N\left(E_{\alpha}\right)$ and $m \in N\left(E_{\beta}\right)$ and suppose that $n m m^{-1} \in N\left(E_{\delta}\right)$. Then

and

$$
n m m^{-1}\left(n m m^{-1}\right)^{-1}=n m m^{-1} m^{-1} n^{-1}=n m m^{-1} n^{-1} \epsilon E_{\delta}
$$

But

$$
\left(n m m^{-1}\right)^{-1} n m m^{-1}=m m^{-1} \cdot n^{-1} n \cdot m m^{-1}=m m^{-1} \cdot n^{-1} n \in E_{8} .
$$

$$
m m^{-1} \cdot n^{-1} n \in E_{\beta} E_{\alpha} \subseteq E_{\gamma},
$$

and so, since the $E_{\alpha}$ are disjoint sets, $\delta=\gamma$.

Now suppose that $n m \in N\left(E_{\epsilon}\right)$. Then

$$
n m(n m)^{-1}=n m m^{-1} n^{-1} \epsilon E_{\epsilon} .
$$

We have already shown that $n m m^{-1} n^{-1} \epsilon E_{\delta}=E_{\gamma}$. Hence $\epsilon=\gamma$ and for any $n \epsilon N\left(E_{\alpha}\right)$ and $m \in N\left(E_{\beta}\right), n m \in N\left(E_{\gamma}\right)$. Since $E_{\alpha} E_{\beta}=E_{\beta} E_{\alpha}$, we also have $m n \in N\left(E_{\gamma}\right)$. This completes the proof of the theorem.

COROLLARY 1. An inverse semigroup is a band of inverse semigroups if and only if it is a semilattice of inverse semigroups. In particular, an inverse semigroup is a band of groups if and only if it is a semilattice of groups.

CoRoLlaRY 2. A semigroup admitting relative inverses in which the idempotents commute is a semilattice of groups.

Proof. This result of Clifford follows immediately from the fact that a semigroup admitting relative inverses is the class sum of disjoint groups [3].

An implication of this result, not noted at the time, is that one of the defining conditions of normal subsemigroups which are unions of groups given in ([1], p. 400) can be omitted. In 
fact, condition (3) in [1] is necessarily satisfied, and, using the notation of [1], $N=\bigcup N_{c}$ is a normal subsemigroup of the inverse semigroup $S$ if and only if, $\uparrow$ for any $a \epsilon S$ and any idempotent $f \in S$,

$$
a N_{f} a^{-1} \subseteq N_{g} \text {, where } g=a f a^{-1} \text {. }
$$

TheOREM 2. Any semigroup which is a semilattice of inverse semigroups is itself an inverse semigroup.

Proof. Let $N$ be a semigroup which is a semilattice of the inverse semigroups $N\left(E_{\alpha}\right)$, where $\alpha \epsilon J$ and $E_{\alpha}$ is the set of idempotents of $N\left(E_{\alpha}\right)$.

Let $a \in N$ and let $x$ be any common solution of the equations $a x a=a$ and $x a x=x$. We know that a solution always exists, for if $a \in N\left(E_{\alpha}\right)$, then there is a unique $x \in N\left(E_{\alpha}\right)$ satisfying these equations. Suppose there is an $x \in N\left(E_{\beta}\right)$ satisfying these equations. Then, since $N$ is a semilattice, $J$ is commutative and $\alpha \beta=\beta \alpha$. Hence

$$
a x a \in N\left(E_{\alpha}\right) N\left(E_{\beta}\right) N\left(E_{\alpha}\right) \subseteq N\left(E_{\alpha \beta}\right) \text {, }
$$

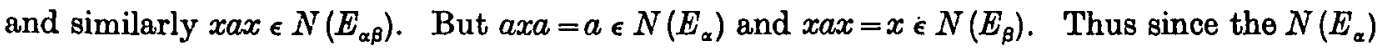
are a set of disjoint semigroups, $\alpha=\alpha \beta=\beta$. Hence $x \in N\left(E_{\alpha}\right)$ and so is unique; so that $N$ is an inverse semigroup.

COROLLARY 1. A semigroup can be the kernel of some homomorphism of an inverse semigroup if and only if it is a semilattice of inverse semigroups.

CoRollary 2. A semigroup which is a semilattice of groups is an inverse semigroup.

3. Structure of semilattices of inverse semigroups. Let $N$ be the class sum of the disjoint inverse semigroups $N\left(E_{\alpha}\right)$, where $\alpha \epsilon J$, and $J$ is a commutative semigroup of idempotents, that is, $J$ is a semilattice. Each $E_{\alpha}$ is the set of idempotents of the corresponding $N\left(E_{\alpha}\right)$. In what follows, for any $\alpha \epsilon J, n_{\alpha}, m_{\alpha}$ will denote arbitrary elements of $N\left(E_{\alpha}\right)$ and $e_{\alpha}, f_{\alpha}, g_{\alpha}$ will denote idempotents, arbitrary except when stated to the contrary, belonging to $E_{\alpha}$.

A right translation of a semigroup $S$ is any mapping $\rho$ of $S$ into $S$ such that for all $s, t \in S,(s t) \rho=s(t \rho)$. A left translation is a mapping $\lambda$ of $S$ into $S$ such that for all $s, t \in S, \lambda(s t)=(\lambda s) t$. Clifford ([8], p. 169) calls the left translation $\lambda$ and the right translation $\rho$ linked in $S$ if, for all $s \in S$,

$$
(\lambda s) \rho=\lambda(s \rho) .
$$

More generally, for any set $S$, we shall say that any two mappings $\lambda, \rho$ of $S$ into $S$, are linked in $S$ if equation (l) holds for all $s \in S$.

We now introduce certain mappings of $N$ into itself, which are linked in the above sense. In terms of these mappings we define a product in $N$, and show that $N$ is a semigroup with respect to this product. It then turns out that the mappings we introduced are left and right translations of this semigroup, and that mappings linked in the set $N$ are translations linked in Clifford's sense in the semigroup $N$.

For all $\alpha \epsilon J$ and each $e_{\alpha} \epsilon E_{\alpha}$, let $\phi\left(e_{\alpha}\right)$ denote a mapping of $N$ into itself which maps each $N\left(E_{\beta}\right)$ into $N\left(E_{\alpha \beta}\right)$ :

$$
n_{\beta} \phi\left(e_{\alpha}\right)=n_{\alpha \beta}
$$

Define in terms of the mappings $\phi$, the mappings $\psi$ of $N$ into itself by

$$
\psi\left(e_{\alpha}\right) n_{\beta}=\left(n_{\beta}^{-1} \phi\left(e_{\alpha}\right)\right)^{-1}
$$

It follows immediately that $\psi\left(e_{\alpha}\right)$ also maps each $N\left(E_{\beta}\right)$ into $N\left(E_{\alpha \beta}\right)$.

† In fact this condition may be further simplified to $a N a^{-1} \subseteq N$ for all $a \in S$. 
Now define a product 0 in the set $N$ by

$$
n_{\alpha} \circ n_{\beta}=\left(n_{\alpha} \phi\left(e_{\beta}\right)\right)\left(\psi\left(f_{\alpha}\right) n_{\beta}\right),
$$

where $e_{\beta}=n_{\beta} n_{\beta}{ }^{-1}$ and $f_{\alpha}=n_{\alpha}{ }^{-1} n_{\alpha}$. The two elements $n_{\alpha} \phi\left(e_{\beta}\right)$ and $\psi\left(f_{\alpha}\right) n_{\beta}$ on the right hand side of (4) both belong to $N\left(E_{\alpha \beta}\right)$ and are considered to be multiplied as in $N\left(E_{\alpha \beta}\right)$.

Suppose that the mappings $\phi$ have the following properties :

P1. $n_{\alpha} \phi\left(e_{\alpha}\right)=n_{\alpha} e_{\alpha}$;

P2. $e_{\alpha} \phi\left(e_{\beta}\right)=e_{\beta} \phi\left(e_{\alpha}\right)$;

P3. each pair of mappings $\psi\left(e_{\alpha}\right), \phi\left(e_{\beta}\right)$ are linked in $N$;

P4. $\phi\left(e_{\alpha}\right) \phi\left(e_{\beta}\right)=\phi\left(e_{\alpha \beta}\right)$, where $e_{\alpha \beta}=\left(e_{\alpha} \phi\left(e_{\beta}\right)\right)\left(e_{\alpha} \phi\left(e_{\beta}\right)\right)^{-1}$;

P5. $\quad\left(n_{\alpha} m_{\alpha}\right) \phi\left(e_{\beta}\right)=\left(n_{\alpha} \phi\left(f_{\alpha \beta}\right)\right)\left(m_{\alpha} \phi\left(e_{\beta}\right)\right)$, where $f_{\alpha \beta}=\left(m_{\alpha} \phi\left(e_{\beta}\right)\right)\left(m_{\alpha} \phi\left(e_{\beta}\right)\right)^{-1}$.

Then, with respect to the operation $\circ, N$ becomes an inverse semigroup which is a semilattice of inverse semigroups, the elements of the semilattice being the $N\left(E_{x}\right)$. It is immediately clear from the property $\mathrm{Pl}$ and the definition of the product $\circ$, that within each $N\left(E_{\alpha}\right)$ the operation o coincides with the multiplication already given in $N\left(E_{\alpha}\right)$. Hence, by Theorem 2, we merely have to show that the binary operation $O$ is associative. This follows from the following fourteen lemmas.

Before proceeding we comment on the meaning of the five properties P1-5 of the mappings $\phi$. Pl identifies each $\phi\left(e_{\alpha}\right)$, restricted to $N\left(E_{\alpha}\right)$, with a definite right translation of $N\left(E_{\alpha}\right)$ into itself. It is easily seen that this property is consistent with properties P2-5. P2 ensures that $e_{\alpha} \circ e_{\beta}$ is an idempotent and that $e_{\alpha} \circ e_{\beta}=e_{\beta} \circ e_{\alpha}$. P3 implies a weak form of the associative law, $e_{\alpha} \circ\left(n_{\beta} \circ e_{\gamma}\right)=\left(e_{\alpha} \circ n_{\beta}\right) \circ e_{\gamma} . \quad \mathrm{P} 4$ entails that the successive application of two of the mappings $\phi$ gives a third mapping $\phi$. P5 turns out to be equivalent to the condition (in the semigroup $N)$ that each $\phi\left(e_{B}\right)$ is a right translation of each $N\left(E_{\alpha}\right)$ within the semigroup $N\left(E_{\alpha}\right) \cup N\left(E_{\alpha \beta}\right)$.

\section{Lemma 1.}

$$
\left(n_{\alpha} \circ n_{\beta}\right)^{-1}=n_{\beta}^{-1} \circ n_{\alpha}^{-1} \text {. }
$$

Proof. This lemma follows immediately from the definition of the operation 0 and the properties of inverses in $N\left(E_{\alpha \beta}\right)$.

LEMMA 2. $\quad e_{\alpha} \circ e_{\beta}=e_{\alpha} \phi\left(e_{\beta}\right)=e_{\beta} \phi\left(e_{\alpha}\right)=\psi\left(e_{\alpha}\right) e_{\beta}=\psi\left(e_{\beta}\right) e_{\alpha}=e_{\beta} \circ e_{\alpha} \epsilon E_{\alpha \beta}$.

Proof. By P3, $\psi\left(e_{\alpha}\right)$ and $\phi\left(e_{\alpha}\right)$ are linked. Thus

$$
\left(\psi\left(e_{\alpha}\right) e_{\beta}\right) \phi\left(e_{\alpha}\right)=\psi\left(e_{\alpha}\right)\left(e_{\beta} \phi\left(e_{\alpha}\right)\right),
$$

so that, using the definition (3) of $\psi\left(e_{\alpha}\right)$, we have

$$
\left(e_{\beta} \phi\left(e_{\alpha}\right)\right)^{-1} \phi\left(e_{\alpha}\right)=\left(\left(e_{\beta} \phi\left(e_{\alpha}\right)\right)^{-1} \phi\left(e_{\alpha}\right)\right)^{-1} \text {. }
$$

An element of $N\left(E_{\alpha \beta}^{\prime}\right)$ equal to its inverse must be idempotent, and so $\left(e_{\beta} \phi\left(e_{\alpha}\right)\right)^{-1} \phi\left(e_{\alpha}\right)$ is idem. potent.

By PI

$$
\psi\left(e_{\alpha}\right) e_{\alpha}=\left(e_{\alpha} \phi\left(e_{\alpha}\right)\right)^{-1}=e_{\alpha}^{-1}=e_{\alpha},
$$

so that again applying P3 to $\psi\left(e_{\alpha}\right)$ and $\phi\left(e_{\beta}\right)$ we have

$$
e_{\alpha} \phi\left(e_{\beta}\right)=\left(\psi\left(e_{\alpha}\right) e_{\alpha}\right) \phi\left(e_{\beta}\right)=\psi\left(e_{\alpha}\right)\left(e_{\alpha} \phi\left(e_{\beta}\right)\right)=\left(\left(e_{\alpha} \phi\left(e_{\beta}\right)\right)^{-1} \phi\left(e_{\alpha}\right)\right)^{-1},
$$

an idempotent, since by $\mathrm{P} 2 e_{\alpha} \phi\left(e_{\beta}\right)=e_{\beta} \phi\left(e_{\alpha}\right)$.

The definition of the operation o together with condition P2 now gives

$$
e_{\alpha} \circ e_{\beta}=\left(e_{\alpha} \phi\left(e_{\beta}\right)\right)\left(\psi\left(e_{\alpha}\right) e_{\beta}\right)=\left(e_{\alpha} \phi\left(e_{\beta}\right)\right)\left(e_{\beta} \phi\left(e_{\alpha}\right)\right)^{-1}=\left(e_{\alpha} \phi\left(e_{\beta}\right)\right)\left(e_{\alpha} \phi\left(e_{\beta}\right)\right)^{-1}=e_{\alpha} \phi\left(e_{\beta}\right)
$$

The remaining equalities asserted in the lemma follow from condition P2 and the definition of the mappings $\psi$. 
Lemma 3. $\quad\left(e_{\alpha} \circ e_{\beta}\right) \circ e_{\gamma}=e_{\alpha} \circ\left(e_{\beta} \circ e_{\gamma}\right)$.

Proof. An application of condition P3 gives

$$
\left(\psi\left(e_{\alpha}\right) e_{\beta}\right) \phi\left(e_{\gamma}\right)=\psi\left(e_{\alpha}\right)\left(e_{\beta} \phi\left(e_{\alpha}\right)\right),
$$

from which, using the results of Lemma 2, Lemma 3 immediately follows.

Lemma 4.

$$
\phi\left(e_{\alpha}\right) \phi\left(e_{\beta}\right)=\phi\left(e_{\alpha} \circ e_{\beta}\right) .
$$

Proof. This follows directly from Lemma 2 and $\mathrm{P} 4$.

Lemma 5.

$$
\begin{aligned}
& n_{\alpha} \circ e_{\beta}=n_{\alpha} \phi\left(e_{\beta}\right) ; \\
& e_{\alpha} \circ n_{\beta}=\psi\left(e_{\alpha}\right) n_{\beta} .
\end{aligned}
$$

Proof. Let $f_{\alpha}=n_{\alpha}^{-1} n_{\alpha}$. From Lemma 4 and Pl we have

$$
n_{\alpha} \phi\left(e_{\beta}\right)=\left(n_{\alpha} f_{\alpha}\right) \phi\left(e_{\beta}\right)=n_{\alpha} \phi\left(f_{\alpha}\right) \phi\left(e_{\beta}\right)=n_{\alpha} \phi\left(f_{\alpha} \circ e_{\beta}\right) \text {. }
$$

On the other hand, since $n_{\alpha} \phi\left(e_{\beta}\right) \in N\left(E_{\alpha \beta}\right), \mathrm{Pl}$ implies that

$$
n_{\alpha} \circ e_{\beta}=\left(n_{\alpha} \phi\left(e_{\beta}\right)\right)\left(f_{\alpha} \circ e_{\beta}\right)=n_{\alpha} \phi\left(e_{\beta}\right) \phi\left(f_{\alpha} \circ e_{\beta}\right) \text {, }
$$

so that, by Lemmas 2,3 and 4 , we have

$$
n_{\alpha} \circ e_{\beta}=n_{\alpha} \phi\left(f_{\alpha} \circ e_{\xi}\right) \text {. }
$$

Hence $n_{\alpha} \circ e_{\beta}=n_{\alpha} \phi\left(e_{\beta}\right)$; and the other equation asserted in the lemma now follows from Lemma 1 .

Lemma 6.

$$
\begin{aligned}
& n_{\alpha} \circ\left(e_{\beta} \circ e_{\gamma}\right)=\left(n_{\alpha} \circ e_{\beta}\right) \circ e_{\gamma} ; \\
& \left(e_{\alpha} \circ e_{\beta}\right) \circ n_{\gamma}=e_{x} \circ\left(e_{\beta} \circ n_{\gamma}\right) .
\end{aligned}
$$

Proof. The first equation here is merely condition P4 restated by means of Lemmas 4 and 5. The second equation then follows by an application of Lemma 1.

LEMMA 7. Let $f_{\alpha}=n_{\alpha}^{-1} n_{\alpha}$ and $e_{\beta}=n_{\beta} n_{\beta}^{-1}$. Then

$$
\left(n_{\alpha} \circ e_{\beta}\right)\left(f_{\alpha} \circ n_{\beta}\right)=\left(n_{\alpha} \circ e_{\beta}\right) \circ n_{\beta}=n_{a} \circ\left(f_{\alpha} \circ n_{\beta}\right)=n_{\alpha} \circ n_{\beta} .
$$

Proof. Lemma 5 implies that

$$
n_{\alpha} \circ n_{\beta}=\left(n_{\alpha} \phi\left(e_{\beta}\right)\right)\left(\psi\left(f_{\alpha}\right) n_{\beta}\right)=\left(n_{\alpha} \circ e_{\beta}\right)\left(f_{\alpha} \circ n_{\beta}\right) .
$$

Hence, applying this result to $\left(n_{\alpha} \circ e_{\beta}\right) \circ n_{\beta}$, we have

$$
\left(n_{x} \circ e_{\beta}\right) \circ n_{\beta}=\left(\left(n_{a} \circ e_{\beta}\right) \circ e_{\beta}\right)\left(\left(\left(e_{\beta} \circ n_{\alpha}^{-1}\right)\left(n_{\alpha} \circ e_{\beta}\right)\right) \circ n_{\beta}\right) .
$$

Since $\left(n_{\alpha} \circ e_{\beta}\right)\left(f_{\alpha} \circ e_{\beta}\right)=n_{\alpha} \circ e_{\beta}$, by Lemma 6 , and multiplication is associative in $N\left(E_{\alpha \beta}\right)$, by another application of Lemma 6 we have

$$
\left(\left(e_{\beta} \circ n_{\alpha}^{-1}\right)\left(n_{\alpha} \circ e_{\beta}\right)\right) \circ n_{\beta}=\left(\left(e_{\beta} \circ n_{\alpha}^{-1}\right)\left(n_{\alpha} \circ e_{\beta}\right)\right)\left(\left(f_{\alpha} \circ e_{\beta}\right) \circ n_{\beta}\right) .
$$

Hence, again using the associativity of multiplication in $N\left(E_{\alpha \beta}\right)$, Lemma 6 implies that

$$
\left(n_{\alpha} \circ e_{\beta}\right) \circ n_{\beta}=\left(n_{\alpha} \circ e_{\beta}\right)\left(f_{\alpha} \circ n_{\beta}\right) \text {. }
$$

The other equation to be proved follows similarly.

Lemma 8. Let $g_{\alpha}=m_{\alpha}^{-1} m_{\alpha}$ and $e_{3}=n_{\beta} n_{\beta}^{-1}$. Then

and

$$
\left(n_{\alpha} m_{x}\right) \circ n_{\beta}=\left(n_{\alpha} m_{\alpha}\right) \circ\left(g_{\alpha} \circ n_{\beta}\right)
$$

Proof. By Lemmas 6 and 7 ,

$\left(n_{\alpha} m_{\alpha}\right) \circ\left(g_{\alpha} \circ n_{\beta}\right)=\left(n_{\alpha} m_{\alpha}\right) \circ\left(\left(m_{\alpha}^{-1} n_{\alpha}^{-1} n_{\alpha} m_{\alpha}\right) \circ\left(g_{\alpha} \circ n_{\beta}\right)\right)=\left(n_{\alpha} m_{\alpha}\right) \circ\left(\left(m_{\alpha}^{-1} n_{\alpha}^{-1} n_{\alpha} m_{\alpha}\right) \circ n_{\beta}\right)$, 
and this, again by Lemma 7, equals $\left(n_{\alpha} m_{\alpha}\right) \circ n_{\beta}$.

The other equation follows similarly.

LeMma 9. Let $g_{\alpha}=m_{\alpha}^{-1} m_{\alpha}$ and $e_{\beta}=n_{\beta} n_{\beta}^{-1}$. Then

and

$$
\begin{aligned}
& \left(n_{\alpha} m_{\alpha}\right) \circ n_{\beta}=\left(\left(n_{\alpha} m_{\alpha}\right) \circ e_{\beta}\right)\left(g_{\alpha} \circ n_{\beta}\right), \\
& n_{\alpha} \circ\left(n_{\beta} m_{\beta}\right)=\left(n_{\alpha} \circ e_{\beta}\right)\left(f_{\alpha} \circ\left(n_{\beta} m_{\beta}\right)\right) .
\end{aligned}
$$

Proof. By applying Lemmas 8 and 6 alternately, we have

$$
\begin{aligned}
\left(n_{\alpha} m_{\alpha}\right) \circ n_{\beta} & =\left(n_{\alpha} m_{\alpha}\right) \circ\left(g_{\alpha} \circ n_{\beta}\right) \\
& =\left(n_{\alpha} m_{\alpha}\right) \circ\left(\left(e_{\beta}^{-} \circ g_{\alpha}\right)\left(g_{\alpha} \circ n_{\beta}\right)\right) \\
& =\left(\left(n_{\alpha} m_{\alpha}\right) \circ\left(e_{\beta} \circ g_{\alpha}\right)\right)\left(\left(e_{\beta} \circ g_{\alpha}\right)\left(g_{\alpha} \circ n_{\beta}\right)\right) \\
& =\left(\left(n_{\alpha} m_{\alpha}\right) \circ e_{\beta}\right)\left(g_{\alpha} \circ n_{\beta}\right) .
\end{aligned}
$$

The other equation follows similarly.

LeMma 10. $\quad\left(n_{\alpha} \circ e_{\beta}\right) \circ n_{\gamma}=n_{\alpha} \circ\left(e_{\beta} \circ n_{\gamma}\right)$.

Proof. Since $\left(n_{\alpha} \circ e_{\beta}\right)=\left(n_{\alpha} \circ e_{\beta}\right)\left(f_{\alpha} \circ e_{\beta}\right)$, where $f_{\alpha}=n_{\alpha}{ }^{-1} n_{\alpha}$, an application of Lemma 8 gives

$$
\left(n_{\alpha} \circ e_{\beta}\right) \circ n_{\gamma}=\left(n_{\alpha} \circ e_{\beta}\right) \circ\left(\left(f_{\alpha} \circ e_{\beta}\right) \circ n_{\gamma}\right) \text {. }
$$

Put $e_{\gamma}=n_{\gamma} n_{\gamma}{ }^{-1}$. Then, since idempotents are combined associatively by Lemma 3, and since

$$
\left(f_{\alpha} \circ e_{\beta}\right) \circ n_{\gamma}=\left(f_{\alpha} \circ e_{\beta} \circ e_{\gamma}\right)\left(\left(f_{\alpha} \circ e_{\beta} \circ e_{\gamma}\right) \circ n_{\gamma}\right),
$$

again by Lemma 8 , we have

$$
\begin{aligned}
\left(n_{\alpha} \circ e_{\beta}\right) \circ n_{\gamma} & =\left(\left(n_{\alpha} \circ e_{\beta}\right) \circ\left(f_{\alpha} \circ e_{\beta} \circ e_{\gamma}\right)\right)\left(\left(f_{\alpha} \circ e_{\beta} \circ e_{\gamma}\right) \circ n_{\gamma}\right) \\
& =\left(n_{\alpha} \circ\left(f_{\alpha} \circ e_{\beta} \circ e_{\gamma}\right)\right)\left(\left(f_{\alpha} \circ e_{\beta} \circ e_{\gamma}\right) \circ n_{\gamma}\right),
\end{aligned}
$$

by Lemma 6 .

A similar argument shows that this latter expression also equals $n_{\alpha} \circ\left(e_{\beta} \circ n_{y}\right)$; from which the result required follows.

LEMma 11.

$$
\left(e_{\alpha} \circ n_{\beta}\right) \circ e_{\gamma}=e_{\alpha} \circ\left(n_{\beta} \circ e_{\gamma}\right) \text {. }
$$

Proof. This is merely a restatement of condition P3, making use of the result of Lemma 5 .

LEMMA 12.

$$
\begin{aligned}
& \left(n_{\alpha} m_{\alpha}\right) \circ e_{\beta}=n_{\alpha} \circ\left(m_{\alpha} \circ e_{\beta}\right) ; \\
& e_{\alpha} \circ\left(n_{\beta} m_{\beta}\right)=\left(e_{\alpha} \circ n_{\beta}\right) \circ m_{\beta} .
\end{aligned}
$$

Proof. Let $m_{\alpha} \circ e_{\beta}=n_{\alpha \beta}$, and $f_{\alpha \beta}=n_{\alpha \beta} n_{\alpha \beta}{ }^{-1}$. Then, by condition P5, using our previous lemmas, $f$ we have

But, by Lemma 10,

$$
\left(n_{\alpha} m_{\alpha}\right) \circ e_{\beta}=\left(n_{\alpha} \circ f_{\alpha \beta}\right)\left(m_{\alpha} \circ e_{\beta}\right)
$$

Hence

$$
\left(n_{\alpha} \circ f_{\alpha \beta}\right)\left(m_{\alpha} \circ e_{\beta}\right)=n_{\alpha} \circ\left(f_{\alpha \beta} \circ\left(m_{\alpha} \circ e_{\beta}\right)\right)=n_{\alpha} \circ\left(m_{\alpha} \circ e_{\beta}\right)
$$

$$
\left(n_{\alpha} m_{\alpha}\right) \circ \dot{e_{\beta}}=n_{\alpha} \circ\left(m_{\alpha} \circ e_{\beta}\right)
$$

and the other equation follows similarly, or from Lemma 1.

LemaA 13. Let $e_{\alpha}=n_{\alpha} n_{\alpha}^{-1}$. Then

$$
n_{\alpha} \circ\left(n_{\alpha}^{-1} \circ e_{\beta}\right)=\left(e_{\beta} \circ n_{\alpha}\right) \circ n_{\alpha}^{-1}=\left(e_{\beta} \circ n_{\alpha}\right)\left(n_{\alpha}^{-1} \circ e_{\beta}\right)=e_{\alpha} \circ e_{\beta} \text {. }
$$

Proof. Lemma 12 gives immediately that

$$
e_{\alpha} \circ e_{\beta}=\left(n_{\alpha} n_{\alpha}^{-1}\right) \circ e_{\beta}=n_{\alpha} \circ\left(n_{\alpha}^{-1} \circ e_{\beta}\right),
$$

† P5 is used only for the proof of Lemma 12. It is easy to see that we need only assume that P5 holds for $\alpha \geqslant \beta$, that is, for $\alpha \beta=\beta$. 
and

$$
e_{\beta} \circ e_{\alpha}=e_{\beta} \circ\left(n_{\alpha} n_{\alpha}^{-1}\right)=\left(e_{\beta} \circ n_{\alpha}\right) \circ n_{\alpha}^{-1} \text {. }
$$

Consider now

$$
\left(\left(e_{\beta} \circ n_{\alpha}\right) \circ n_{\alpha}^{-1}\right) \circ e_{\beta}=\left(\left(e_{\beta} \circ n_{\alpha}\right)\left(f_{\alpha \beta} \circ n_{\alpha}^{-1}\right)\right) \circ e_{\beta},
$$

where $f_{\alpha \beta}=\left(n_{\alpha}^{-1} \circ e_{\beta}\right)\left(e_{\beta} \circ n_{\alpha}\right)$. Because $e_{\beta} \circ n_{\alpha}$ and $f_{\alpha \beta} \circ n_{\alpha}^{-1}$ both belong to $N\left(E_{\alpha \beta}\right)$, we may apply Lemma 12 to obtain $\dagger$

$$
\left(\left(e_{\beta} \circ n_{\alpha}\right)\left(f_{\alpha \beta} \circ n_{x}{ }^{-1}\right)\right) \circ e_{\beta}=\left(e_{\beta} \circ n_{\alpha}\right)\left(\left(f_{\alpha \beta} \circ n_{\alpha}{ }^{-1}\right) \circ e_{\beta}\right) \text {. }
$$

But, bylLemma 11,

from the definition of $f_{\alpha \beta}$.

$$
\left(f_{\alpha \beta} \circ n_{\alpha}^{-1}\right) \circ e_{\beta}=f_{\alpha \beta} \circ\left(n_{\alpha}^{-1} \circ e_{\beta}\right)=n_{\alpha}^{-1} \circ e_{\beta},
$$

Hence, finally, we have

$$
e_{\alpha} \circ e_{\beta}=\left(\left(e_{\beta} \circ n_{\alpha}\right) \circ n_{\alpha}^{-1}\right) \circ e_{\beta}=\left(e_{\beta} \circ n_{\alpha}\right)\left(n_{\alpha}^{-1} \circ e_{\beta}\right),
$$

which completes the proof of the lemma.

LEMIMa 14.

$$
\left(n_{\alpha} \circ n_{\beta}\right) \circ n_{\gamma}=n_{\alpha} \circ\left(n_{\beta} \circ n_{\gamma}\right) .
$$

Proof. Let $f_{\alpha}=n_{\alpha}{ }^{-1} n_{\alpha}, e_{\beta}=n_{\beta} n_{\beta}^{-1}$ and $e_{\gamma}=n_{\gamma} n_{\gamma}{ }^{-1}$. Since $n_{\alpha} \circ n_{\beta}=\left(n_{\alpha} \circ e_{\beta}\right)\left(f_{\alpha} \circ n_{\beta}\right)$, an application of Lemma 9 gives

$$
\left(n_{\alpha} \circ n_{\beta}\right) \circ n_{\gamma}=\left(\left(n_{\alpha} \circ n_{\beta}\right) \circ e_{\gamma}\right)\left(\left(\left(n_{\beta}^{-1} \circ f_{\alpha}\right)\left(f_{\alpha} \circ n_{\beta}\right)\right) \circ n_{\gamma}\right) .
$$

Consider now $\left(n_{\alpha} \circ n_{\beta}\right) \circ e_{\gamma}$. By Lemma 12, we have

$$
\begin{aligned}
\left(n_{\alpha} \circ n_{\beta}\right) \circ e_{\gamma} & =\left(n_{\alpha} \circ e_{\beta}\right) \circ\left(\left(f_{\alpha} \circ n_{\beta}\right) \circ e_{\gamma}\right) \\
& =\left(\left(n_{\alpha} \circ e_{\beta}\right) \circ\left(\left(\left(f_{\alpha} \circ n_{\beta}\right) \circ e_{\gamma}\right)\left(e_{\gamma} \circ\left(n_{\beta}^{-1} \circ f_{\alpha}\right)\right)\right)\right)\left(\left(f_{\alpha} \circ n_{\beta}\right) \circ e_{\gamma}\right) .
\end{aligned}
$$

By Lemmas 11 and 13, however,

$$
\begin{aligned}
\left(\left(f_{\alpha} \circ n_{\beta}\right) \circ e_{\gamma}\right)\left(e_{\gamma} \circ\left(n_{\beta}^{-1} \circ f_{\alpha}\right)\right) & =\left(f_{\alpha} \circ\left(n_{\beta} \circ e_{\gamma}\right)\right)\left(\left(e_{\gamma} \circ n_{\beta}^{-1}\right) \circ f_{\alpha}\right) \\
& =f_{\alpha} \circ\left(\left(n_{\beta} \circ e_{\gamma}\right)\left(e_{\gamma} \circ n_{\beta}^{-1}\right)\right) .
\end{aligned}
$$

Hence $\left(n_{\alpha} \circ e_{\beta}\right) \circ\left(\left(\left(f_{\alpha} \circ n_{\beta}\right) \circ e_{\gamma}\right)\left(e_{\gamma} \circ\left(n_{\beta}^{-1} \circ f_{\alpha}\right)\right)\right)=\left(n_{\alpha} \circ e_{\beta}\right) \circ\left(f_{\alpha} \circ\left(\left(n_{\beta} \circ e_{\gamma}\right)\left(e_{\gamma} \circ n_{\beta}^{-1}\right)\right)\right)$

$$
=\left(n_{\alpha} \circ f_{\alpha}\right) \circ\left(e_{\beta} \circ\left(\left(n_{\beta} \circ e_{\gamma}\right)\left(e_{\gamma} \circ n_{\beta}^{-1}\right)\right)\right) \text {, }
$$

by Lemmas 6, 3 and 2 .

But $n_{\alpha} \circ f_{\alpha}=n_{\alpha}$ and, by Lemmas 12 and 11 ,

$$
e_{\beta} \circ\left(\left(n_{\beta} \circ e_{\gamma}\right)\left(e_{\gamma} \circ n_{\beta}^{-1}\right)\right)=\left(n_{\beta} \circ e_{\gamma}\right)\left(e_{\gamma} \circ n_{\beta}^{-1}\right) \text {. }
$$

Combining these results gives

$$
\left(n_{\alpha} \circ n_{\beta}\right) \circ e_{\gamma}=\left(n_{\alpha} \circ\left(\left(n_{\beta} \circ e_{\gamma}\right)\left(e_{\gamma} \circ n_{\beta}^{-1}\right)\right)\right)\left(\left(f_{\alpha} \circ n_{\beta}\right) \circ e_{\gamma}\right) .
$$

Hence we have that

where

$$
\left(n_{\alpha} \circ n_{\beta}\right) \circ n_{\gamma}=\left(n_{\alpha \beta \gamma}\left(\left(f_{\alpha} \circ n_{\beta}\right) \circ e_{\gamma}\right)\right) m_{\alpha \beta \gamma},
$$

and

$$
\begin{aligned}
n_{\alpha \beta \gamma} & =n_{\alpha} \circ\left(\left(n_{\beta} \circ e_{\gamma}\right)\left(e_{y} \circ n_{\beta}^{-1}\right)\right) \\
m_{\alpha \beta \gamma} & =\left(\left(n_{\beta}^{-1} \circ f_{\alpha}\right)\left(f_{\alpha} \circ n_{\beta}\right)\right) \circ n_{y} .
\end{aligned}
$$

A similar argument now shows that

$$
n_{\alpha} \circ\left(n_{\beta} \circ n_{y}\right)=n_{\alpha \beta \gamma}\left(\left(f_{\alpha} \circ\left(n_{\beta} \circ e_{\gamma}\right)\right) m_{\alpha \beta \gamma}\right) .
$$

The lemma now follows from the associative law in $N\left(E_{\alpha \beta \gamma}\right)$ and from Lemma 11.

This completes the proof that $N$ is a semigroup which is a semilattice of the inverse semigroups $N\left(E_{\alpha}\right)$.

† This result may be obtained without using Lemma 12 or condition P5. 
Conversely, let $N$ be any semigroup which is a semilattice of disjoint inverse semigroups $N\left(E_{\alpha}\right)$. Then, since by Theorem $2, N$ is an inverse semigroup, if we define the mapping $\phi\left(e_{\alpha}\right)$ by the equation

$$
n_{\beta} \phi\left(e_{\alpha}\right)=n_{\beta} e_{\alpha}
$$

and the mapping $\psi\left(e_{\alpha}\right)$ by the equation

$$
\psi\left(e_{\alpha}\right) n_{\beta}=\left(n_{\beta}{ }^{-1} \phi\left(e_{\alpha}\right)\right)^{-1}=e_{\alpha} n_{\beta},
$$

$\phi\left(e_{\alpha}\right)$ and $\psi\left(e_{\alpha}\right)$ map each $N\left(E_{\beta}\right)$ into $N\left(E_{\alpha \beta}\right)$. It is then easy to see that properties P1-5 are valid for these mappings $\phi$, and that the operation o defined by (4) coincides with the multiplication already given in $N$.

Finally, consider two isomorphic semigroups $N, N^{\prime}$ which are both semilattices of inverse semigroups. The semilattice $J$ of $N$ and its elements $N\left(E_{\alpha}\right)$ determine a set of isomorphic inverse subsemigroups $\bar{N}\left(\bar{E}_{\bar{\alpha}}\right)$ of $N^{\prime}$ which form a semilattice $\bar{J}$ isomorphic to $J$. Further, the right translations $\phi\left(e_{\alpha}\right)$ of $N$ determine right translations $\bar{\phi}\left(\bar{e}_{\bar{\alpha}}\right)$ of $N^{\prime}$ such that, in the isomorphic mapping between $N$ and $N^{\prime}$, when $n_{\beta} \leftrightarrow \bar{n}_{\bar{\beta}}$, then

$$
n_{\beta} \phi\left(e_{\alpha}\right) \longleftrightarrow \bar{n}_{\bar{\beta}} \bar{\phi}\left(\bar{e}_{\bar{\alpha}}\right) .
$$

Conversely, the semilattice $J^{\prime}$ formed by the inverse subsemigroups $N^{\prime}\left(E_{\alpha^{\prime}}^{\prime}\right)$ of $N^{\prime}$ and the right translations $\phi^{\prime}\left(e_{\alpha^{\prime}}^{\prime}\right)$ of $N^{\prime}$ determine an isomorphic semilattice of isomorphic subsemigroups of $N$ and right translations of $N$ such that the equation corresponding to (5) is satisfied. It follows therefore that the set of all sets consisting of (i) a semilattice $J$ determined by $N$, (ii) the inverse semigroups $N\left(E_{\alpha}\right)$ which are the elements of $J$ and (iii) the set of right translations $\phi\left(e_{\alpha}\right)$ satisfying relations (2) and (3) and properties P1-5, is a set of invariants under isomorphism of the semigroup $N$. On the other hand it is clear that if two semigroups $N, N^{\prime}$ have the same set of invariants in the above sense, then $N$ is isomorphic to $N^{\prime}$. In fact, any semilattice $J$ with associated inverse semigroups $N\left(E_{\alpha}\right)$ and mappings $\phi\left(e_{\alpha}\right)$ determines $N$ to within isomorphism. Thus our set of invariants provides a complete set of invariants for $N$.

We note that if $N$ is isomorphic to $N^{\prime}$ and $J$ is isomorphic to $J^{\prime}$, then the corresponding $N\left(E_{\alpha}\right)$ and $N^{\prime}\left(E_{\alpha^{\prime}}^{\prime}\right)$ determined by the isomorphism between $J$ and $J^{\prime}$ are not necessarily isomorphic. For let $N$ be a semigroup consisting of the three commuting idempotents $e, f, g$, and such that $e f=e, e g=e, f g=f$. Then $N$ is a semilattice with elements $N_{\alpha}=(e, f)$ and $N_{\beta}=(g)$, where $\alpha \beta=\alpha$. It is also a semilattice consisting of $N_{\alpha^{\prime}}=(e)$, and $N_{\beta^{\prime}}=(f, g)$, where $\alpha^{\prime} \beta^{\prime}=\alpha^{\prime}$. Then $\alpha \longleftrightarrow \alpha^{\prime}, \beta \leftrightarrow \beta^{\prime}$ is an isomorphism of $J=(\alpha, \beta)$ onto $J^{\prime}=\left(\alpha^{\prime}, \beta^{\prime}\right)$. But $N_{\alpha}$ and $N_{\alpha^{\prime}}$ are not isomorphic.

When $N$ and $N^{\prime}$ are restricted to being semilattices of groups the situation is simpler. It is then clear that if $N$ is isomorphic to $N^{\prime}$, then $J$ is necessarily isomorphic to $J^{\prime}$ and the groups which correspond under this isomorphism are also isomorphic in pairs. The complete set of invariants consists therefore in this case of merely one semilattice $J$, the set of groups which are the elements of $J$ and the set of right translations $\phi$.

We summarise the results of this section in the following theorem.

TheOrem 3. Let $N$ be the class sum of the disjoint inverse semigroups $N\left(E_{\alpha}\right)$, where $E_{\alpha}$ is the set of idempotents of $N\left(E_{\alpha}\right)$ and $\alpha$ belongs to the semilattice $J$. Define mappings $\phi$ of $N$ into itself by equation (2) and suppose that the mappings $\phi$ have properties P1-5.

Then if we define a product $\circ$ in the class $N$ by means of equation (4), $N$ becomes a semigroup which is a semilattice of the inverse semigroups $N\left(E_{\alpha}\right) . N$ is necessarily an inverse semigroup. 
The semilattice $J$, the inverse semigroups $N\left(E_{\alpha}\right)$ and the mappings $\phi$ determine $N$ to within isomorphism.

Conversely, any semilattice of inverse semigroups has a structure determined in the above manner.

The set of all sets of semilattices $J$ of inverse semigroups $N\left(E_{\alpha}\right)$ and the corresponding mappings $\phi$ associated as above with $N$ forms a set of complete invariants for $N$.

\section{REFERENCES}

1. G. B. Preston, Inverse semi-groups, London Math. Soc., 29 (1954), 396-403.

2. A. H. Clifford, Bands of semigroups, Proc. Amer. Math. Soc. 5 (1954), 499-504.

3. A. H. Clifford, Semigroups admitting relative inverses, Ann. of Math., 42 (1941), 1037-1049.

4. A. E. Liber, K teorii obobščnnyh grupp, Dokl. Akad. Nauk. SSSR, 97 (1954), 25-28.

5. R. Croisot, Demi-groupes inversifs et demi-groupes réunions de demi-groupes simples, Ann. de L'École Norm., (3) 70 (1953), 361-379.

6. W. D. Munn and R. Penrose, A note on inverse semigroups, Proc. Cambridge Phil. Soc. 51 (1955), 396-399.

7. V. V. Vagner, Obobščennye gruppy, Dokl. Akad. Nauk. SSSR, 84 (1952), 1119-1122.

8. A. H. Clifford, Extensions of semigroups, Trans. Amer. Math. Soc. 68 (1950), 165-173.

Royal Mritary College of Science

ShrIVENHAM

\section{ON THE CLOSED GRAPH THEOREM \\ by ALEX. P. ROBERTSON and WENDY ROBERTSON \\ (Received 8th September, 1956)}

The closed graph theorem is one of the deeper results in the theory of Banach spaces and one of the richest in its applications to functional analysis. This note contains an extension of the theorem to certain classes of topological vector spaces. For the most part, we use the terminology and notation of $\mathrm{N}$. Bourbaki [1], contracting " locally convex topological vector space over the real or complex field " to " convex space "; here we confine ourselves to convex spaces.

Suppose that $E$ is a separated (i.e. Hausdorff) convex space and that its dual $E^{\prime}$ has the weak topology $\sigma\left(E^{\prime}, E\right)$. Then $E$ is called fully complete if a vector subspace $M^{\prime}$ of $E^{\prime}$ is closed whenever $M^{\prime} \cap U^{0}$ is closed for every neighbourhood $U$ of the origin in $E$. A fully complete space is complete; a closed vector subspace of a fully complete space is fully complete and so also is a quotient by a closed vector subspace (H. S. Collins [2]). Any Fréchet space is fully complete (J. Dieudonné and L. Schwartz [3], Théorème 5, Corollaire). There are other fully complete spaces; for example the algebraic dual $E^{*}$ of any vector space $E$ is fully complete under the topology $\sigma\left(E^{*}, E\right)$ (Collins, [2], Corollary 17.2). It is not difficult to show that the dual $E^{\prime}$ of a Fréchet space $E$ is fully complete under any topology between the topology of compact convergence and the Mackey topology $\tau\left(E^{\prime}, E\right)$; in particular the strong dual of a reflexive Fréchet space is fully complete. 